\title{
REVISIÓN/REVIEW
}

\section{LA ENSEÑANZA DE LA HISTORIA EN VENEZUELA: UNA APROXIMACIÓN A SU COMPRENSIÓN COMO FENÓMENO}

María Elena Del Valle de Villalba': Universidad Metropolitana (Caracas). Venezuela.

manedelvalle@gmail.com

\section{RESUMEN}

El presente artículo compila una revisión de las principales características de la enseñanza de la Historia en Venezuela, en Latinoamérica y algunos países de Europa. El mismo se propone una descripción de las principales prácticas pedagógicas empleadas en la administración de los contenidos históricos y una valoración a sus posibles efectos.

PALABRAS CLAVE: Enseñanza de la Historia - Didáctica - Ciencias Sociales

\footnotetext{
${ }^{1}$ María Elena Del Valle de Villalba: Universidad Metropolitana (Caracas). Venezuela. 


\title{
THE TEACHING OF HISTORY IN VENEZUELA: AN APPROACH TO UNDERSTANDING AS PHENOMENON
}

\begin{abstract}
This article compiles an overview of the main features of the teaching of history in Venezuela, Latin America and some countries in Europe. The same is proposed description of the pedagogical practices employed in the historical content and an assessment of their possible effects.
\end{abstract}

KEY WORDS: History Teaching - Teaching - Social Sciences

\section{INTRODUCCIÓN}

La cuestión sobre la enseñanza de la Historia, los textos y manuales escolares usados en su enseñanza y el discurso de los docentes como mediadores será el tópico a abordar en las siguientes líneas. Para ello, se ha dividido este aparte en tres para describir los antecedentes de cada uno de los aspectos señalados:

1.- Estudios precedentes sobre el análisis del discurso en el aula de Historia.

2.- Estudios sobre el análisis de textos escolares.

3.- Estudios sobre la interacción docente-alumno.

\section{ESTUDIOS PRECEDENTES SOBRE EL ANÁLISIS DEL DISCURSO EN EL AULA}

Briolo, J y Herrera, H. (2003) realizan un proyecto de investigación denominado "La comunicación del conocimiento científico en las clases de física de la EGB 3 y Polimodal en escuelas de Catamarca", a cargo de un equipo interdisciplinario de docentes de las Facultades de Ciencias Exactas y Humanidades. El corpus del trabajo estuvo formado por una muestra de clases grabadas y transcriptas, complementadas con notas de campo tomadas por los docentes observadores y con registros en video. El estudio realizado por los investigadores parte del presupuesto que considera a la educación como un proceso de carácter social, proceso que se desarrolla en un marco específico determinado por parámetros históricos y culturales, protagonizado por actores con roles definidos de acuerdo con los grupos a los que pertenecen y que, además, mantienen una relación jerárquica asimétrica, razonamientos que sirven de antecedente a nuestra investigación.

Asimismo, se concibe a la relación docente - alumno, en el contexto áulico, como una interacción fundamentalmente lingüística, ya que la transmisión de información, la construcción del conocimiento, la evaluación de los aprendizajes, en suma todas las interacciones áulicas son posibles solo a través de la lengua. Por esta razón el abordaje realizado, así como las conclusiones a las que llegaron son un camino recorrido que servirá para orientar nuestra investigación. 
Jarquín, G. (2006) plantea en su estudio que el programa de Bachillerato Internacional propone que cada institución educativa construya su propio plan de lectura y ejes temáticos así como el desarrollo de dinámicas particulares dentro del aula, a partir de los cuales los estudiantes van a experimentar la literatura durante dos años. El sistema de evaluación que presenta el programa, esbozado en un grupo de manuales y folletos, corresponde a un currículum, si por este se entiende el diseño de las estrategias de evaluación, así como los componentes que deben ser analizados en el acercamiento al mundo literario.

En este sentido afirma que el vacío que parecen sentir los profesores y las profesoras, es el de una metodología o estrategia formalizada para el análisis de tales componentes, lo cual degenera en la ausencia en ocasiones de cualquier tipo de interacción entre docente y alumno.

La costumbre de los estudiantes de asumir una receta para la comprensión de un texto, no es gratuita, ha sido bien aprendida y transmitida por el cuerpo docente, pues ofrece mayor seguridad saber que uno más uno es dos, sin asumir el riesgo que tal vez uno más uno no sea ni dos ni cuatro ni ningún número. En este estudio la autora describe la dinámica comunicacional generada en el aula de clases no cual nos servirá de guía para el establecimiento de categorías de análisis y elementos a observar.

Uno de los trabajos revisados para determinar el estado de la cuestión en este aparte, tiene su soporte en las investigaciones de Peter Jhon. El autor plantea además de la preocupación en relación a los contenidos que se enseñan en la escuela básica, critica el hecho de que se ha pasado por alto uno de los factores más importantes que es a su juicio, el tipo de conocimiento que necesitan los profesores para enseñar Historia.

Desde su perspectiva, sostiene que los profesores de Historia deben ser capaces no solo de definir para sí mismos y para el alumnado que los oye las verdades aceptadas en la materia, sino que también deben ser capaces de explicar porque una determinada proposición debe ser considerada como valiosa y relevante y como esta se relaciona con otras informaciones que están fuera del Delta de la disciplina histórica.

Otro trabajo interesante es el de F. Audigier et al (1994) en el cual analizan los cruces entre áreas del saber semejantes. Afirman los autores que el debate político introduce algunas variables en el discurso dentro del aula generando de esta manera dinámicas antes no consideradas. Por otra parte señalan que el labor del docente hacer accesible a los alumnos el discurso a veces hermético de los libros de texto.

Weiss (1978) señala en un informe sobre la enseñanza de las Ciencias Sociales en los Estados Unidos que las estrategias más usadas en la administración de dichas ciencias estaba con frecuencia limitada a la exposición docente y las pruebas periódicas. Otro trabajo que puede considerarse como antecedente de los estudios etnográficos en el campo de la enseñanza de la Historia es el de Stake y Easley (1978) en el cual se llega a importantes conclusiones entre ellas: 
- $\quad$ Se encontró que la manera de enseñar Historia variaba en un profesor a otro.

- Proporcionó importantes informaciones en torno a la relación entre las actividades propuestas por el docente y la dinámica generada en clases.

- La investigación reveló que la enseñanza de la Historia más que estar influenciada por el libro de texto lo está por el profesor de la materia.

Downe y Levstik (1991) en este mismo orden de ideas llegan a las siguientes conclusiones:

- El panorama de los estudios relacionados con la dinámica generada dentro del aula al enseñar Historia es desalentador.

- $\quad$ Son pocos los estudios que se han detenido a describir e interpretar la dinámica generada como producto de la interacción entre el docente y el alumno y de qué manera esta determina la comprensión de los contenidos desarrollados en el aula.

- $\quad$ En la misma perspectiva también señalaron que existe la necesidad de analizar el uso que dan los profesores dentro del aula a la bibliografía, al periódico, la ficción y de qué manera estas permean formas narrativas de enseñanza que puedan motivar y despertar el interés en el alumnado con respecto a los contenidos históricos.

Audigier (1984) realizó una investigación orientada a indagar las diferencias entre las opiniones de los docentes de Historia y Geografía en relación a la pedagogía utilizada en su enseñanza. Para ello se utilizó un cuestionario con preguntas relativas a esas disciplinas. Dichos cuestionarios se aplicaron a 1400 docentes y del análisis de los resultados se obtuvieron las siguientes conclusiones:

- Más del $50 \%$ del profesorado coincide en que enseñar a los alumnos a pensar es su propósito principal.

- $\quad$ El 35\% de los profesores es partidario de impartir conocimientos disciplinares básicos.

- $\quad$ El $60 \%$ de los docentes afirmó priorizar las necesidades del alumno por encima de las demandadas por el programa.

- $\quad 93 \%$ de los docentes expresó que aprobaban las discusiones en el aula como estrategia para el desarrollo de los contenidos.

Blanch (2008) afirma en este sentido que no es habitual que los historiadores y las historiadoras universitarios piensen en la formación de su alumnado como profesores de enseñanza secundaria lo que tal vez explicaría la ausencia de estrategias más motivantes en la administración de los contenidos. Ni tan siquiera, continúa el autor, que tengan inquietudes y preocupaciones didácticas. Son pocos los profesores y profesoras de historia que creen que existe un campo de problemas, y una disciplina, que tiene por objetivo investigar sobre la enseñanza y el aprendizaje de la historia en cualquier contexto educativo p156.

Blanch (2008) acota en su estudio que muchos docentes que imparten esta materia creen poseer las experticias didácticas sin haber sido formados para ellas. A este respecto afirma: Sigue habiendo profesores y profesoras, políticos y ministras, que creen que para enseñar es suficiente con saber aquello que debe enseñarse.

Para enseñar matemáticas basta con saber matemáticas, de la misma manera que para enseñar historia basta con saber historia. Si eso fuera así, sería difícil explicar porqué existen tantos problemas en el aprendizaje de la historia -o de cualquier otra 
materia -, porqué existen tantos jóvenes y adultos que no saben historia o saben muy poca cuando quienes les enseñan han acreditado sus saberes en la Universidad p 156. En el mismo estudio Blanch (2008) propone una lista de aspectos que deben tomarse en consideración al analizar la interacción el aula de Historia así como los aspectos que deberían considerarse en el perfil del comunicador/docente que está en ella:

"... en cualquier acto comunicativo hay que tener en cuenta: a) la formación para la comunicación, la adecuación de lo que se comunica, del discurso, al medio y al contexto, el aprendizaje de determinadas herramientas, etc. Hay que formar al comunicador para que conozca las características y los medios de la comunicación educativa; b) las personas a las que se les comunica algo, con las que se establece algún tipo de comunicación, en nuestro caso los alumnos y las alumnas de secundaria, su predisposición ante lo que les queremos comunicar, sus propósitos y sus intereses para aprender aquello que les comunicamos; y c) lo que se comunica, este caso el conocimiento histórico, y cómo debemos comunicarlo para obtener aprendizajes. Y, además, el contexto en el que se realiza la comunicación, contexto que incluye desde las finalidades o propósitos educativos que la administración educativa otorga a los saberes escolares, el contexto social y cultural, hasta la institución en la que se realiza y la organización espacio-temporal en la que tiene lugar" p 157.

El problema que involucra la enseñanza de cualquier ciencia y en este caso de la Historia es complejo y multidimensional. Según el autor, si se quiere realmente profundizar en la comprensión del fenómeno se debe obligatoriamente analizar este entramado de discursos múltiples.

Jiménez, Pilar y Díaz (2003) en su trabajo Discurso de aula y argumentación: Cuestiones teóricas y Metodológicas, realizan una reflexión en torno al discurso en el aula y su contribución a los procesos de aprendizaje de las ciencias sociales. En su estudio analizan el razonamiento argumentativo usado por los actores: alumnos y docentes, con ejemplos tomados del proyecto denominado RODA (Razonamiento, discusión, argumentación) llevado a cabo en la Universidad de Santiago de Compostela, España.

Con respecto al tipo de investigación que para su comprensión utilizaron en el estudio y recomiendan para quien quiera emprender uno semejante afirman que por su carácter de estudios de caso, las investigaciones sobre discurso del aula son adecuadas para la investigación- acción, estudios en los que los docentes analizan su propia práctica en el aula, actuando como profesores investigadores.

Para el análisis de la argumentación usada por docentes y alumnos generan en su estudio una interesante lista de preguntas:

¿Qué cuenta como dato, como comprobación para las y los actores? 
- ¿ ¿Qué cuenta como conclusión o explicación teórica?

- ¿ ¿Qué cuenta como justificación en apoyo de una conclusión?

- ¿ ¿En qué situaciones utilizan justificaciones los estudiantes?

- $\quad$ ¿Qué tipos de justificaciones?

- ¿Cómo se relacionan las justificaciones con el conocimiento básico que las respalda?

- ¿ ¿Qué proceso siguen para argumentar mientras están resolviendo un problema?

Estas preguntas pueden eventualmente seguir de guía para la observación en interpretación de lo que los autores denominan en su estudio como: Discurso Escolar. Así mismo presentan una clasificación de los esquemas argumentales usados en el aula, generando dos clasificaciones:

Retóricos, razones para convencer al auditorio, frente a dialógicos, que examinan distintas alternativas, siendo éstos los de mayor interés para el análisis del discurso.

- Racionales, que buscan una solución racional a un problema determinado, frente a persuasivos, que pretenden llegar a un consenso p 362.

Adicionalmente, generan un esquema que sirve de guía para el análisis del discurso generado en el aula por docente y alumnos. Los autores sugieren que se interpreten las interacciones en función de:

1) Secuencias de actividades o fases en una unidad didáctica.

2) Secuencias de episodios, por ejemplo, en cada sesión de una unidad y ejes cronológicos (timelines).

3) Secuencias de argumentos a lo largo de una o varias sesiones de trabajo, como las estudiadas durante la identificación de muestras desconocidas con el microscopio $p$ 363.

Giraldo (2009) realiza un estudio en el que describe los actos de habla que predominan en el discurso de los profesores de Educación física, y cuáles de éstos estimulan la creatividad de los estudiantes. El estudio se basa en el análisis del discurso de 14 docentes en 10 colegios públicos y privados de Bogotá D.C. En este estudio concluye que el discurso de los maestros analizados se caracteriza por tener una fuerte expresión en actos de habla expositivos y autoritarios, predominando la dimensión instructiva.

Afirma que al no presentarse un equilibrio de la dimensión instructiva frente a las dimensiones afectiva, emotiva y social, aparecen en ellas de manera débil las valoraciones positivas, la creación de estados de ánimo hacia la experimentación, el ejercicio de la libertad de experimentación y goce del cuerpo, la curiosidad y el trabajo en equipo, que son categorías esenciales para desarrollar la creatividad. Concluye que los actos de habla que estimulan la creatividad motriz de los estudiantes no se presentan con insistencia y regularidad en el discurso del maestro.

En su estudio establece cómo el discurso dentro del aula regula las interacciones 
docente-alumno, orienta la manera de conocer, de sentir y de vivir del alumno. Analiza en su trabajo los llamados Actos de Habla, categoría establecida por Searle y Vanderveken (1985) . En dicha teoría los actos de habla son clasificados en cinco tipos básicos:

- $\quad$ Asertivos: cuando su propósito es representar un estado de cosas como real.

- Compromisivos: su objeto es comprometer al hablante con un curso de acción futuro.

- $\quad$ Directivos: su objeto es comprometer al oyente con un curso de acción futura.

- $\quad$ Declarativos: su propósitos es crear una situación nueva.

- $\quad$ Expresivos: sirven para manifestar sentimientos y actitudes del hablante.

Añade además, la propuesta de Martínez Otero, para quien el discurso docente está constituido por dimensiones interdependientes como la instructiva, afectiva, motivadora y social. Todas estas categorías de análisis del discurso docente que arrojó este trabajo nos servirán de guía para el establecimiento de las nuestras.

Torrealba (2004) en su trabajo La interacción docente y el discurso pedagógico de la ironía, establece que el análisis de las relaciones interpersonales en contextos educativos lleva a considerar que una parte de la efectividad didáctica del discurso docente se basa en la regulación de las relaciones de poder y de distancia que caracterizan las situaciones de clase.

Campagnucci et al (2002) realizan un análisis en torno a tres tipos de relaciones: Docente-Contenido, Docente-Alumno, Alumno-Contenido a través de la aplicación de una "guía de observación de clase". De la aplicación de este instrumento surgieron datos cuantitativos y cualitativos de un total de 40 guías de observación de clases. Asimismo, de sus conclusiones podemos extraer abordajes metodológicos y herramientas de análisis pertinentes para nuestro estudio.

Candela (2001) en su trabajo Corrientes Teóricas sobre discurso en el aula, hace una revisión de las corrientes teóricas que aportan estudios del discurso en el aula. En él expone un recorrido sobre el surgimiento, los aportes y algunos cuestionamientos, desde disciplinas afines, de enfoques como la lingüística, la sociolinguística, la etnografía y la etnometodología. En este trabajo se propone estudiar la relación entre discurso y procesos educativos en la escuela, asumiéndolo como un proceso público de negociación y creación cultural.

González (2006) en su trabajo Análisis del discurso en el aula: una herramienta para nuestra autoevaluación, se plantean mostrar como los profesores pueden empezar a reflexionar con el fin de conocer mejor qué sucede en sus clases. En su estudio se propone analizar la necesidad de tomar conciencia de que existen sistemas de análisis y de reflexión sobre la actuación didáctica de los docentes, que aunque puedan parecer laboriosos al principio, pueden reportar grandes beneficios a la actividad docente.

Briolo y Herrera (2003) en una ponencia presentada en el Congreso Regional de 
Ciencia y Tecnología en la Universidad de Catamarca, Argentina, plantean el análisis de las intenciones del docente y la progresión en el discurso áulico. En este trabajo, el corpus está conformado por una muestra de clases grabadas y transcriptas, complementadas con notas de campo tomadas por los docentes observadores y con registros en video. Este estudio parte del presupuesto que considera a la educación como un proceso de carácter social, proceso que se desarrolla en un marco específico determinado por parámetros históricos y culturales, protagonizado a su vez por actores con roles definidos.

Asimismo, conciben la relación docente-alumno en el contexto áulico, como una interacción fundamentalmente lingüística, ya que a su juicio la transmisión de la información, la construcción del conocimiento, la evaluación de los aprendizajes obtenidos solo son posibles a través del uso de la lengua.

Llobera (1990) en su artículo Reconsideración del discurso interactivo, reivindica el papel didáctica del discurso en el aula. Desde su perspectiva, en los últimos años se ha enfatizado demasiado en la presentación de los materiales didácticos, en perjuicio del discurso generado dentro del aula. Plantea en su trabajo una serie de hipótesis interesantes para nuestro estudio como por ejemplo:

- $\quad$ Que el discurso en el aula, a pesar de sus peculiaridades, presenta muchas de las características que presenta el discurso en las interacciones sociales generales.

- $\quad$ Es la exposición de estas características lo que permite al alumno construir sus propias hipótesis sobre las reglas del uso del discurso.

- $\quad$ Es la actuación en la interacción la que permite la construcción de un discurso significativo en la clase.

Y por último que

- $\quad$ La construcción de un discurso significativo parece ser condición básica para el aprendizaje $\mathrm{p} 67$.

Álvarez (2005) en su artículo Contornos de entonación en el discurso de una docente. Su relación con algunos aspectos pragmáticos, describe los aspectos entonacionales que pueden ser observados en el discurso de una docente dentro del aula con el objeto de determinar algunos aspectos pragmáticos relevantes de ese discurso como diversos actos de habla usados para aseverar, dar órdenes, pedidos, sugerencias y realizar distintas clases de preguntas.

Tarabay y León (2007) en su artículo La argumentación como forma de comunicación en el discurso del profesor universitario, analizan las estrategias argumentativas de los profesores de ciencias sociales en sus clases magistrales. Del repertorio de estrategias usadas por los docentes los investigadores examinaron las que regulan la acción comunicativa. El artículo defiende la idea de que la clase magistral, pese a su reconocida limitación, tiene un carácter eminentemente dialógico y constituye un ritual de celebración del saber.

En este artículo los investigadores generan categorías de análisis que sirven de base para el análisis del discurso del docente dentro del aula de clase. 
Viladot, Gómez y Malagarriga (2008) realizan en su artículo Análisis del Discurso en un aula de música, un estudio de las interacciones en el aula como medio para comprender el proceso enseñanza-aprendizaje. En este trabajo se analiza una conversación dentro de un aula de música y se identifican las estrategias discursivas que ayudan a comprender el proceso de construcción conjunta de significados. En este caso la investigación se llevó a cabo a través de la observación directa no participante, anotaciones de aula, entrevistas a la profesora y una muestra de cinco alumnos, grabación en audio y video de las diez sesiones de clases, abordaje que servirá de guía para el análisis de las interacciones en el aula de clases.

Cabrera (2003) en su trabajo Discurso en el aula, estudia usando un enfoque etnográfico, el tipo de comunicación predominante en el aula. Para ello observó la comunicación verbal y no verbal del docente y sus alumnos y alumnas en el aula escolar, además de las percepciones que éste tiene respecto a su práctica. En su trabajo establece tipos de comunicación, las cuales define como: Afectiva, Autoritaria, Conciliadora, Flexible, Jerárquica analizando no solo la del docente sino la de los alumnos deteniéndose en la verbal y en la no verbal. La comunicación no verbal es clasificada por el autor como: Kinésica, Paralinguística y Proxémica, clasificaciones que nos servirán de guía para observar las interacciones en el aula y los tipos de lenguaje usado.

De Longhi (2000) en su estudio El Discurso del profesor y del alumno: análisis didáctico, contribuyen a la comprensión de los procesos de construcción de significados y conocimiento que emergen gracias a un uso específico del lenguaje dentro del aula de clases. En su trabajo analiza en particular las preguntas que realiza el docente en su discurso y las respuestas que genera el estudiante a las mismas.

Otro trabajo interesante es el de Guanipa y Velazco (2005) en el que analizan la hermenéutica del discurso profesoral en el contexto cognitivo de los estudiantes. El estudio realizado se ubicó en el área de las ciencias humanas, fue de modalidad de campo contando con una muestra constituida por 347 estudiantes, 72 profesores y 10 coordinadores de área. En este trabajo se concluyó que la función traductora del mensaje del profesor ofrece de manera deficiente la comprensión del discurso a los estudiantes, pues este no es inteligible en su contexto cognitivo.

Martínez-Otero (2004) en su trabajo La calidad del discurso educativo: Análisis y regulación a través de un modelo pentadimensional, ofrece un modelo psicopedagógico que permite analizar la potencia formativa del discurso a través de cinco dimensiones interdependientes: instructiva, afectiva, motivadora, social y ética. A partir del modelo que ofrece en su estudio establece tipologías del profesorado y del alumnado que sirven de referencia para el análisis que pretendemos realizar en esta investigación.

Gvirtz, Larripa y Oría (2005) en su trabajo Prácticas discursivas pedagógicas, didácticas y escolares: algunas categorías para repensar la relación entre el saber y la escuela, revisan las nociones de discurso pedagógico o discurso escolar además de indagar los procesos a través de los cuales los diversos saberes se transforman dentro 
del aula.

Por último Walter et al. (2008) realizan una exhaustivo análisis del discurso del docente como elemento mediador para el fomento del aprendizaje significativo de los estudiantes en los procesos evaluativos. En análisis del discurso lo realizan en función de categorías emergentes de los datos. Los resultados obtenidos por los investigadores muestran algunas características del discurso del profesor que dificultan el fomento de niveles de aprendizaje de orden superior en el estudiante.

\section{ESTUDIOS PRECEDENTES SOBRE EL ANÁLISIS DE TEXTOS ESCOLARES}

Ramírez, T. (2002) en su trabajo El texto escolar como objeto de investigación analiza el papel que cumple el texto escolar en el proceso enseñanza-aprendizaje, como mediador entre el docente y el alumno y su papel como recurso pedagógico de singular relevancia. En este trabajo se logran dos propósitos, en primer término inventariar y considerar los diferentes términos y significados usados para definir este medio de enseñanza, con el objeto de proponer un concepto que permita contribuir a acabar con la anarquía terminológica y en segundo término revalorizar al texto escolar como objeto de investigación, dada su importancia estratégica en el proceso de enseñanza-aprendizaje.

Braslavsky (2007) En su artículo Los Libros de Texto en su contexto: Argentina 19751989, realiza la descripción del estado del arte sobre los libros de texto para la enseñanza de la Historia en la Argentina, caracteriza el sistema educativo argentino y el papel que cumplen los textos de Historia en el nivel medio, además de realizar una revisión de los programas oficiales para la enseñanza de la Historia. En su trabajo concluye que la investigación sobre los libros de textos en la Argentina es sin duda necesaria, se deben continuar haciendo precisiones y proponiendo alternativas. Se debe a su juicio procurar que llegue a la escuela más de la producción historiográfica argentina.

Monroy (1993) en su trabajo Análisis educativo del texto escolar, realiza un análisis de la dimensión educativa y pedagógica del texto escolar, aborda los usos y consumo de los mismos, la organización didáctica de su contenido y las especificidades de estos en el contexto del sistema educativo colombiano. En su estudio llega a conclusiones como:

- $\quad$ El maestro depende del texto para enseñar.

- $\quad$ Los profesores dependen de los textos y en muchos casos no tienen tiempo para preparar las clases, volviéndose este su principal apoyo.

Por último se plantea si existe verdadera autonomía del profesor, por su solvencia intelectual para acompañar en el proceso de conocimiento que emprende el alumno en el desarrollo de la cátedra.

Mendoza, (2006) en su artículo La perspectiva discursiva en el análisis historiográfico del libro escolar, aborda las posibilidades teórico-metodológicas que brinda la semántica al análisis del discurso de los libros escolares, en particular a los libros de 
textos de Historia. En su investigación responde a preguntas como: ¿Cómo se construye el relato en los textos escolares?, ¿Cuál es la lógica discursiva de la periodización de la Historia en estos discursos?, ¿Cuál es el orden y los niveles del discurso narrativo? Desentrañando el sentido del devenir histórico que el lector construye a través de los textos.

Bernete, (1994) aporta en su trabajo Cómo analizar las representaciones sociales en los libros de texto de Historia, una metodología específica para analizar las representaciones de grupos nacionales contenidas en los libros de texto de Historia, contemplados en cuanto a productos comunicativos cuando intervienen en la enculturización de los estudiantes y que, pueden ser fuente de prejuicios. En su investigación desmonta conceptos como: distinción etnocéntrica, distinción prejuiciosa y distinción estereotípica, abordando además el concepto de nación, objetivando los contenidos latentes de determinados productos culturales.

Bralich (1990) en su texto Los textos escolares como instrumento ideológico, determina los contenidos que a su juicio pueden tener una carga intencionada que direccione a los estudiantes hacia una corriente de pensamiento en particular. En su obra analiza los textos de diversas editoriales del Uruguay desmontando su construcción en función a la categoría: valores llegando a conclusiones muy valiosas para la investigación que emprendo.

En su tesis de grado para optar al título de Licenciado en Historia, Romero, (1994) plantea preguntas interesantes en torno a las temáticas desarrolladas en los textos escolares chilenos y las posiciones ideológicas y/o políticas de los autores de dichos textos. De esta manera el autor intenta desmontar las posibles intenciones que un autor puede tener al estructurar el discurso a través del cual transmite los contenidos históricos.

De igual forma, define la enseñanza de la Historia como una herramienta de socialización en los valores de la cultura dominante, un modelador de ciudadanía y de las fidelidades con respecto a lo que se considera histórico o no y los libros de textos son el medio más fiel a través del cual se puede analizar el proceso antes descrito.

Braslavsky (1993) en su obra Los usos de la Historia en los libros de texto para escuelas primarias argentinas (1916-1930), realiza la revisión de casi cien libros de texto entre 1853 y 1930 usados en las escuelas primarias argentinas. En su análisis trabaja el uso de los mitos en lo que denomina "la creación de una épica patriótica desteñida, deslucida y aburrida" p 56. Los mitos usados en la transmisión de los saberes históricos son a su juicio dos: el de los orígenes, dispositivo para integrar a los niños a un presente común a partir de un pasado compartible y el del progreso indefinido de una nación grande y privilegiada.

Eiros (1996) en su artículo Los estudiantes y los textos históricos: una relación conflictiva, centra su interés en el análisis de los textos de Historia señalando en ellos los modelos historiográficos y la metodología de enseñanza empleada en ellos. Analiza en su estudio los niveles de comprensión de los alumnos clasificándolos en: 
preliminar, básico, competencia mínima, estudios posteriores y avanzado.

Cubas, (1996) en artículo Contenido ideológico de los Textos Escolares Primarios de Historia Nacional (1890-1910) llega a las siguientes conclusiones:

- $\quad$ Los textos escolares son fuente importante para el análisis de valores, actitudes e ideologías de un momento histórico determinado.

- $\quad$ Llega a establecer la corriente historiográfica a la que pertenece el texto.

- Logra determinar la influencia de las principales ideologías en la construcción de los textos.

- $\quad$ Destaca la construcción de mitos y la exaltación de los símbolos patrios.

- Señala la didáctica usada en los textos describiendo las estrategias usadas por los autores de los textos.

Maristany (1988) en su trabajo titulado Las Ficciones del pasado en los manuales de Historia argentina (1880-1910), analiza el proceso de formación y consolidación de los conceptos de estado-nación a través de los textos escolares. Afirma que es a través de ellos que pueden analizarse los paradigmas fundantes de la nación argentina así como también el culto a los personajes históricos como elemento característico de idiosincrasia argentina, elemento que desde su perspectiva se va cultivando desde el texto escolar.

Muscará (1999) realiza en su trabajo titulado Contenido ideológico de los textos escolares, un exhaustivo acercamiento a tres manuales escolares, a los que define como el espacio donde nos encontramos no sólo con las opiniones e ideas de sus autores sino también con canales de comunicación de las ideas de la sociedad y en los que podremos percibir cuál es el proceso de transformación que se produce desde el momento en que se formula una teoría. En su estudio analiza el libro del escolar de Pablo Pizzurno, Sentimiento de José María Aubin, La Patria de José Manuel Eizaguirre, abordando su contenido en función de: el análisis del contenido del texto y datos biográficos del autor. Kauffmann (2001) en su artículo Producciones sobre textos escolares argentinos: hitos, tendencias y potencialidades, tiene por objeto perfilar el estado del arte de las producciones abocadas al estudio de los textos escolares en la Argentina hasta diciembre del año 2001. En su artículo señala importantes conclusiones entre las que podemos mencionar:

La mayoría de los artículos se han centrado en el análisis historiográfico sobre el contenido de los manuales, la estructura didáctica de los textos, la distancia entre la producción académica y el contenido, los aspectos dogmáticos y las orientaciones político/ideológicas de los manuales escolares.

Realiza un apartado para describir aquellos artículos que abordan la temática de los textos escolares centrándose en las cuestiones de producción, circulación y difusión de los mismos.

Grinberg y Palermo, (2000) en su trabajo Mujeres y trabajo en los textos escolares: crisis y perspectivas, abordan las formas que asume el trabajo de varones y mujeres en los textos escolares de los noventa incorporando la noción de significado del 
trabajo para su análisis desde un enfoque de género. La incorporación de esta categoría de análisis le permite otorgar a la reflexión que realizan una nueva lógica, en tanto este concepto se refiere a un proceso de construcción socio-históricamente situado. De esta manera, a lo largo del trabajo intentan brindar algunas respuestas a las siguientes interrogantes:

- ¿ ¿Qué ha pasado en los libros destinados a la enseñanza a partir de la década del ochenta?

- $\quad$ ¿De qué manera se presenta la imagen de la mujer en los textos?

- $\quad$ ¿Cómo afectan a la presentación del trabajo femenino la crisis que atraviesa el mundo del trabajo?

En su estudio las investigadoras intentan dar respuesta a algunas de estas preguntas deteniéndose en la forma de concebir el trabajo de varones y mujeres en este fin de siglo. La manera como emergen en este estudio las categorías de análisis y el abordaje metodológico empleado sirve de orientación para el estudio emprendido.

Souto (2002) investigador de la Universidad de Barcelona, en su trabajo titulado Los manuales escolares y su influencia en la instrucción escolar, realiza el análisis de los manuales producidos en España, Portugal y América Latina durante el período 1808 a 1990. En su investigación define al libro escolar como una herramienta que se utiliza en la mayoría de las ocasiones como el eje de la programación didáctica de una clase. Explica los contextos legales y teóricos en los cuales se produce la edición de los manuales escolares y afirma que el poder político discrimina en muchos casos a unos en beneficio de otros, señalando de la misma manera que se debe contar con las características propias de la difusión de este tipo de productos: amistades entre autores y profesores, el prestigio adquirido, las redes comerciales, etc.

Galván (2000) realiza en el Seminario: Libros Escolares Mexicanos, siglos XVIII, XIX y $\mathrm{XX}$, la compilación de las reflexiones y las principales conclusiones a la que se llegó. Entre las más importantes podemos mencionar:

- Ha surgido una nueva historiografía de la educación, en la que el libro como objeto de estudio juega un papel protagónico, una historiografía que vuelve a los libros de texto, a los textos escolares, como fuente principal, ya que representan un modo de concebir y practicar la enseñanza.

- $\quad$ Se considera a los libros o manuales escolares como instrumentos ideológicos o de poder, dirigido a los jóvenes susceptibles de ser manipulados y a la vez poco críticos.

- $\quad$ Se sostiene que los textos escolares constituyen instrumento de numerosos intereses e intenciones, pero que también son sujetos de abundantes intervenciones y regulaciones.

Morales, y Lischinsky (2008) en su artículo Discriminación a través de las ilustraciones de los libros de texto de Educación Secundaria Obligatoria en España, realizan un análisis socio-semiótico del racismo discursivo, tal como se presenta en textos complejos que combinan lo visual y lo verbal. A partir de un corpus de 250 imágenes 
obtenidas de diez libros de ciencias sociales de nivel medio publicados en España entre 1995 y 2004, exploran cómo la ideología dominante produce representaciones diferenciadas de un endogrupo y un exogrupo en los sub-sistemas visual y lingüístico. De la misma manera, examinan cómo dentro de las construcciones de elevado control del género discursivo los valores, estereotipos, ideologías y significados que no pueden expresarse de manera verbal se comunican visualmente. Llegan a la importante conclusión de que en los libros de texto la selección de imágenes no es neutra ni arbitraria, sino que intenta reproducir el sistema de desigualdades, caracterizado por la discriminación, el racismo y la xenofobia. Esta investigación nos sugirió el análisis no solo de los contenidos verbales, sino también de los no verbales en los libros de texto.

García, (2000) en su producción titulada Textos Escolares y Patrimonio Cultural, se propuso observar cómo se trata el tema del patrimonio cultural y su conservación en la educación escolar chilena. Para ello realizó durante el año 2000 una revisión de los textos escolares de ese año, con los cuales estudian gran parte de los niños chilenos desde Primer Año hasta Cuarto Año de Enseñanza Media. Adicionalmente se consideraron en este estudio los programas de estudio del Ministerio de Educación llegando a las siguientes conclusiones:

- En los primeros años de educación básica se imparten a los niños conocimientos generales de las tradiciones nacionales, la diversidad cultural y el conocimiento de su entorno natural y material.

-Después de la revisión de los textos para la educación media se observó que sólo en el texto de Ciencias Sociales se trata el tema de patrimonio cultural.

-En el programa de estudio para el segundo año medio se busca que los estudiantes desarrollen una visión global acerca del desenvolvimiento de la historia nacional y comprendan que la realidad actual tiene sus antecedentes en los procesos históricos que la han ido conformando.

Por último, y en relación a la categoría fundamental a observar en su investigación, afirman que el tema de Patrimonio Cultural no está considerado en los programas de estudio como un aspecto relevante para la historia de Chile, ni dentro de los temas que se tratan en los textos y programas de estudio para el curso de Lenguaje y Comunicación que de alguna manera está vinculado a la cultura nacional a través de la literatura y distintos medios de comunicación.

Valls, (2001) en su publicación Los nuevos retos de las investigaciones sobre los manuales escolares de Historia: entre textos y contextos, establece que la investigación de los libros de texto ha de orientarse no solo a la revisión de su contenido, sino al ámbito socio-educativo donde se inscriben. Afirma que la idea de mejorar los manuales de Historia implica primeramente plantearse las condiciones del proceso enseñanza-aprendizaje y sus referentes teórico-prácticos en un marco de complejidad y diversidad conceptual.

Según su visión, ya no es suficiente abordar el estudio de los manuales escolares desde una concepción restrictiva de los mismos, sino que los manuales escolares 
exigen ser considerados necesariamente en su relación tanto con los alumnos como con los docentes y que ambas relaciones son profundamente complejas y que son diversas en función de la disciplina escolar de la que se trate.

Fontaine y Eyzaguirre, (1997) en su trabajo ¿Por qué es importante el texto escolar? Realiza afirmaciones importantes que constituyen importante antecedente de esta investigación, entre las cuales podemos citar:

- $\quad$ No sirve un sistema educacional aunque cumpla con los objetivos que se ha trazado si estos son demasiado pobres o irrelevantes para satisfacer las necesidades individuales y de la sociedad en general. La capacidad de compensar las desigualdades de entrada de sus alumnos es también componente importante de la calidad de un sistema.

- Las investigaciones más recientes sobre el impacto de políticas educacionales indican que, las siguientes formas de intervención del sistema escolar tienen más posibilidades de afectar positivamente el aprendizaje: mejorar el currículum, aumentar el material de instrucción, aumentar el tiempo de instrucción, mejorar la calidad de la instrucción considerando como la más importante la calidad de la instrucción.

- $\quad$ Demuestran también que los alumnos aprenden más usando textos de estudio que sin ellos, que el uso frecuente de los textos se correlacionan significativamente con un mayor número de conceptos aprendidos.

Los textos de estudio no solo "transportan" el currículum sino también, en gran medida, lo determinan. Corresponde entonces a los textos especificar y decidir por qué medios lograr estos objetivos, que alcance y volumen tendrán los contenidos, que nivel de dificultad se exigirá p 358.

Tiana, (1999) en su artículo La Investigación Histórica sobre los manuales escolares en España, establece que una de las fuentes históricas de la que se dispone para realizar historia de la educación es la constituida por el conjunto de los libros utilizados en las instituciones educativas. Con unas características $u$ otras, con uno u otro formato, con tales o cuales contenidos, los manuales se han convertido en un instrumento central en los procesos de enseñanza y aprendizaje que tiene lugar en el ámbito escolar.

Ossenbach,(2000) en su artículo La Investigación sobre los manuales escolares en América Latina, señala que junto a las investigaciones sobre cuestiones de actualidad, el uso de los libros de texto como fuente histórica ha ido cobrando una creciente relevancia a medida que la investigación histórico-educativa se ha ido consolidando. Los trabajos que se han realizado en relación con los libros escolares han abordado prioritariamente, como lo ha hecho también la investigación histórico-educativa en general, su influencia en la creación de las identidades nacionales y los imaginarios colectivos, así como en los procesos de secularización de los diversos países latinoamericanos a partir de la independencia.

Alarcón y Conde (2003) identifican y catalogan manuales escolares, analizan desde la perspectiva histórica, las condiciones de producción, circulación y el uso del libro o manual escolar en un espacio regional definido, teniendo en cuenta los tres niveles del 
contexto social en medio del cual se ha desenvuelto a través del tiempo la cultura escolar. Dividieron su investigación en dos partes, la primera: a partir de una identificación previa, elaboran un censo guía de los textos, manuales y libros escolares que circularon entre 1832 y 1898.

De estos realizan la recopilación sistemática de todos aquellos documentos que de una u otra manera se relacionan con la circulación y uso de estas publicaciones. Analizan las disposiciones legales de carácter educativo, planes de estudio, programas, pruebas, pasquines, proclamas y folletos. La segunda parte: intenta lograr una mirada inter-disciplinaria del objeto de estudio, en lo que se refiere a los contenidos temáticos, características bibliográficas, editoriales, curriculares, políticas, ideológicas, imaginarios, representaciones sociales y políticas, así como la receptividad de los lectores de los libros escolares.

Simancas (1998) afirma que el texto escolar generalmente es estudiado como recurso de aprendizaje, pero a su juicio va mucho más allá ya que los docentes tienden a trabajar más con él que con el programa institucional. La labor escolar a su parecer se realiza sobre o en relación al libro de texto. El manual se ha convertido, en la mayoría de los casos, en la única fuente de consulta y guía pedagógica de alumnos y profesores. En su trabajo examina, desde la perspectiva curricular, como el libro de texto traduce y codifica la cultura seleccionada en el curriculum. En su estudio genera una serie de Items que pueden orientar la evaluación de los textos escolares entre los cuales es pertinente destacar:

- $\quad$ Revisar el temario-índice para determinar las estructuras conceptuales en las que se organiza el contenido; lo que se incluye y lo que se excluye.

- Revisar las fuentes de información para establecer si se explicitan; si son diversas; si la información está cerrada o se presenta abierta para ser completada o modificada.

- Ubicar el contenido dentro de los ámbitos culturales de selección: conocimiento científico y/o cotidiano; si parte de la estructura de las disciplina o de otro criterio.

- Identificar su organización: modular (unidades globalizadas) o por unidades temáticas, jerarquizadas o en espiral (p85).

En relación con las estrategias didácticas usadas en el texto, también generan algunos indicadores que sin duda servirán de guía:

Analizar las actividades que el texto incluye para lo cual se pueden revisar los ejercicios al final de la unidad.

- Establecer si el texto se adopta al nivel, posibilidades e intereses de los alumnos; si sugiere actividades comunes para el conjunto de la clase; si estimulan el desarrollo psicológico de los estudiantes; si incentivan el desarrollo del lenguaje; si contribuyen a la comprensión del proceso de comprensión de conocimientos.

- $\quad$ Determinar la estructura y el estilo de las tareas.

- Determinar si el texto propone actividades de evaluación: establecer su naturaleza; si estimula la metacognición (p. 86)

Por último, proporcionan algunos atributos en los cuales detenernos en relación con lo que denomina: modelo de profesionalidad docente implícito en el texto.

- $\quad$ Establecer si al profesor se le da información sobre los criterios de elaboración 
del texto; si contiene una guía didáctica para el profesor.

- $\quad$ Reconocer el grado de autonomía del profesor: si puede elegir entre varias actividades y/o materiales distintos; si puede adaptar o introducir nuevas ideas al texto; si le asigna un rol activo o pasivo en el trabajo con el libro y los alumnos; si sugiere competencias docentes relacionadas con la investigación y la búsqueda de nuevas fuentes de información ( $p$ 87)

Ramírez, T. (2007) realiza un profundo análisis de las principales características que revisten los textos escolares desde 1958 hasta el año 2004. En su trabajo describe como se ha ido realizando un desplazamiento de la importancia adjudicada al texto escolar como elemento estratégico para la conformación del sistema educativo. Describe como de su producción y distribución gratuita pasó en épocas más recientes a convertirse en un producto sin supervisión didáctica ni regulación. Los señalamientos que aporta su estudio nos servirá de antecedente para el abordaje de los textos escolares en el área de Historia de Venezuela.

Graterol, A. (2002) en su trabajo El discurso escolar de la ciudadanía: una mirada desde la hermenéutica, realiza un análisis de la definición del señalado constructo en un corpus de textos escolares seleccionados. En su investigación promueve la idea de que los discursos contenidos en los textos constitucionales y en los documentos de la educación formal y, en particular los del área de Ciencias Sociales en el nivel de Educación Básica, están inmersos en contextos sociales y culturales estructurados, por lo que un análisis textual de los códigos y símbolos que lo constituyen deben enfocarse al estudio de la construcción significativa y de la contextualización del Discurso Escolar de la Ciudadanía en la Educación Básica. Este estudio nos suministra algunas orientaciones para el establecimiento de categorías de análisis así como la revisión de las conclusiones a las que llega la investigadora.

Por otra parte, Domínguez, M y Varela, C (2008) realizan un estudio en el que pretenden mostrar cómo un nuevo enfoque de las técnicas tradicionales de análisis textual permite examinar las relaciones que se establecen entre los vocablos utilizados en textos de ciencias para expresar un determinado concepto, así como evidenciar y constatar errores conceptuales o relaciones inadecuadas que propicien su formación.

Para ello aplican el programa informático PAFE (Programa de Análisis de Frecuencias y Entornos) a textos de ciencias de enseñanza básica que versan sobre el Sistema Solar y se establecen comparaciones entre los resultados y las dificultades de enseñanzaaprendizaje encontradas en otras investigaciones para explicar determinados fenómenos astronómicos. El sistema usado para observar las ocurrencias temáticas y discursivas servirá de orientación para la realización de nuestro estudio.

Por su parte Linuesa, M. (2006) en su trabajo Los sistemas de valores en los textos escolares: un modelo de análisis. La autora señala que los contenidos educativos, como elemento configurador del currículo, son susceptibles de varios niveles de análisis: psicopedagógico, epistemológico e ideológico. En su trabajo se centran en algún aspecto de este último nivel.

En primer lugar, define el concepto de «ideología», como forma de conocimiento junto 
al conocimiento científico; asimismo sus implicaciones educativas, que pueden venir manifiestas en múltiples instancias una de las cuales es la escuela y dentro de ésta los contenidos educativos. Luego, analiza el problema metodológico que presenta el análisis de este tipo de mensajes ideológicos. En su estudio solo se ocupa de los textos escritos. Por otro lado, señala que las peculiaridades del conocimiento ideológico impone ciertas condiciones en el uso de determinados modelos. Presenta, por último, una alternativa específica: el método de Actantes de Greimas, enriquecido por la perspectiva que, en el nivel teórico, ofrece la gramática generativa.

Herrera, Pinilla y Suaza (2003) sostienen que en los textos escolares subyacen modelos pedagógicos y que en ellos pueden analizarse aspectos relacionados con el ideal de ser humano que se pretende formar, según la idea de sociedad o de proyecto social al que ellos son articulados. Abordan en su estudio los modelos pedagógicos que circularon en Colombia durante la primera mitad del siglo XX. Clasifican las tendencias en modelos pedagógicos en:

- Escuela Memorística tradicional.

- La Tendencia Pestalozzi: la cual hace énfasis en la intuición y la experiencia de los niños como fuente de conocimiento.

- Tendencia experimental de Wundt.

- Decroly y su consideración de las necesidades sociales del ser humano.

- J. Dewey: con un contenido más social y político, enfatizando la educación como elemento que consolida las democracias.

Afirman que en Colombia los textos y los autores miran al niño de varias maneras y expresan diferentes concepciones. La concepción pre-moderna los ubica como adultos en pequeño, que no tienen uso de razón y conocimiento del deber, con una mente vacía que debe ser llenada.

Bini et al (1977) generan dos categorías esenciales para realizar el análisis de los textos escolares:

El análisis externo: considera al texto como objetos de consumo y describe exhaustivamente su producción y comercialización a través de:

a) Relevamiento de las editoriales que publican libros de texto.

b) Tirada y reediciones.

c) Costos y comercialización: precios de costo, precios de venta, distribución y bocas de expendio.

d) Carácter autoral o anónimo (equipos) de las publicaciones.

Y el análisis interno que implica la descripción de los tres códigos manifiestos que estructuran las obras analizadas: diagramación, ilustración y discurso lingüístico) y el código ideológico subyacente que involucra a los tres manifiestos. Estas categorías sirven de guía para emprender el análisis de los textos.

Tiana (2000) afirma en su trabajo El libro escolar, reflejo de intenciones políticas e 
influencias pedagógicas, que las imágenes pueden constituir una categoría de análisis. A este respecto señala que las ilustraciones en los manuales constituyen uno de los campos de mayor interés para la comprensión de la compleja red de influencias que interactúan en la construcción del imaginario colectivo. Señala, que la inclusión de las imágenes en los textos nunca es arbitraria, puesto que siempre depende de criterios pedagógicos y científicos y que además debe tomarse en cuenta que desde el punto de vista político-ideológico, nunca es neutra.

Argibay, Celorio y Celorio (1991) generan lo que denominan un Modelo Didáctico, este modelo intenta hacer explícitos los diversos elementos que actúan en el proceso didáctico y que se deben tomar en cuenta para situar el papel del libro de texto.

En primer término establecen un: Marco Social.

Lo denominan medio social, generalmente este actúa como globalmente sobre el marco institucional del sistema de enseñanza pero también y al mismo tiempo sobre los agentes y espacios concretos de ejercicio de la enseñanza (profesor, estudiantes, recursos y medios didácticos).

En un segundo nivel, se analiza la imagen proyectada por los medios de comunicación de masas. Estos medios no solamente estructuran una imagen y un sistema de explicación e interpretación de modelo de desarrollo sino que generan y socializan todo un sistema de valores.

En un tercer nivel: los agentes que impugnan el conjunto del modelo, elementos culturales, ideológicos y artísticos.

En segundo término: el Marco Institucional. Este sistema, cuyas características y necesidades propias le conceden cierta independencia, está compuesto por:

El Profesor, que a su juicio genera:

- $\quad$ La construcción del conocimiento histórico-social.

- $\quad$ La ideología, complejo sistema según el cual los individuos ordenan su visión del mundo.

- $\quad$ La percepción de la realidad social. Del profesor, el individuo recibe un aluvión de información que debe digerir y amoldar a sus estructuras previas.

- $\quad$ El profesor supone qué puede interesarles, hasta dónde puede llegar con ellos, qué es relevante y qué es importante, cuál puede ser su grado de respuesta a un determinado tipo de acción, qué grado de conocimientos y estructuras previas poseen, etc

Y por último:

El Estudiante: concebido como agente educativo y receptor del proceso de enseñanza. Se le asume como un elemento portador de supuestos previos que pueden ser explicitados en la acción didáctica o, por el contrario, quedar ocultos. Las mencionadas 
dimensiones y categorías sirven, sin duda de guía para el análisis que emprendemos.

Otro elemento en el que se detienen los investigadores en relación a los libros de texto, es el abordaje de categorías específicas como la planteada por Werz (2000). En su producción Reflexiones sobre la imagen de Bolívar y la enseñanza de la Historia de Venezuela, señala que los textos escolares presentan a Bolívar como un héroe nacional y latinoamericano de importancia histórica mundial, generando en este caso un nuevo atributo en el cual detenernos: Los héroes o actores en la historia y su abordaje.

En ese mismo orden de ideas, Galván (2005) afirma que los libros de texto, en el caso específico de México, "no solo muestran a los héroes, sino que también permiten que, ciertos invisibles se vayan convirtiendo en visibles" p 205. La imagen de los héroes y antihéroes forman a su juicio imaginarios colectivos y en el caso concreto de la Historia mexicana "los imaginarios marcan aquello de los podemos agarrarnos, aquello que nos puede sostener" p 226.

Por último, como antecedente para esta categoría de análisis esta el trabajo de Chiroque, S. (2007) quien aborda en los textos escolares de Perú el tratamiento realizado a juicio a Fujimori. Todos los textos revisados por el autor, han sido distribuidos en los últimos años, en fecha posterior al segundo Gobierno de Fujimori. El análisis realizado se detiene en los mensajes explícitos; sin ingresar a un análisis semiótico que ciertamente es importante. El abordaje metodológico y el sistema utilizado en este estudio nos suministrarán indicios para realizar el nuestro.

\section{ESTUDIOS SOBRE LA INTERACCIÓN ENTRE EL DOCENTE Y EL ALUMNO DENTRO DEL AULA}

Kalafunga, M y Obeleagu, A. (2002) realizan un análisis de la interacción que se genera dentro del aula en las escuelas primarias de Tanzania. En este estudio se examina la interacción de los niños en el marco de las actividades pedagógicas y extracurriculares en las escuelas.

Para saber cómo se producen, oponen, reproducen y legitiman las experiencias humanas durante el proceso de enseñanza en la clase se detuvieron en los siguientes puntos:

- Las características de la interacción entre docentes y alumnos (niñas y varones) en el aula.

- Las opiniones de los docentes sobre el papel de varones y niñas en la vida escolar.

- El tipo de tareas que los padres y docentes encargan a varones y niñas en el hogar y en la escuela.

- Los juegos y las actividades de esparcimiento para los niños.

- Los aspectos de la escolaridad que no satisfacen a los alumnos. Todos los aspectos señalados servirán de guía para la elaboración de los cuestionarios para la observación de las clases.

En segundo término, cabe destacar el estudio de Torrealba, M. (2004) quien es su 
trabajo La interacción docente y el discurso pedagógico de la ironía. La autora afirma que discurso pedagógico se refiere a sujetos que hablan y actúan en el mundo de la vida escolar, con expresiones y acciones susceptibles de ser comprendidas. Plantea que el punto de partida es este terreno en el que se produce la articulación de la pedagogía como forma de saber. La comprensión de la epísteme pedagógica, de sus condiciones de posibilidad, y lo propio de sus prácticas discursivas, sus maneras de decir, pensar y actuar; constituyen el terreno de cultivo.

Para la autora, este punto de partida, implica reflexionar sobre este y otros modos de subjetivación-comunicativa-pedagógica, se apoya justamente en la fuerza de la palabra, o fuerza retórica, y la persuasión responsable del interlocutor, de allí que contenga alguna forma de oposición, una argumentación diferente, diálogo que involucra muchas veces un enfrentamiento, otros tejidos de significación. Dada las implicaciones y alcances de su estudio, el mismo es considerado como antecedente de nuestra investigación.

Por último, Sánchez, E (2008) en su trabajo de investigación Elementos para analizar la interacción entre estudiantes y profesores, examina qué ocurre cuando se consideran distintas unidades de análisis y diferentes dimensiones en el análisis de interacciones entre alumnos y profesores durante el desarrollo de las tareas escolares. El autor distingue cinco unidades de análisis -ciclos, episodios, actividades típicas de aula, sesiones y unidades curriculares (enumeradas de menor a mayor amplitud)- y tres dimensiones o focos: los contenidos elaborados -la dimensión Qué, para la cual toma como referencia modelos cognitivos de las tareas desarrolladas-, los patrones de discurso y estructuras de participación que regulan la actividad social y mental de alumnos y profesores -la dimensión Cómo, que para el autor tiene su origen en una concepción sociocultural- y el nivel de autonomía asumido por los alumnos -la dimensión Quién, que plantea emplear a la vez ambos marcos teóricos. El abordaje realizado por este investigador es estructurado y sistemático lo cual sirve de guía tanto en aspectos teóricos como metodológicos.

Busani (2006) analiza en su trabajo los significados -acerca del cuerpo/sujeto- que aparecen naturalizados en el discurso de los estudiantes. Interpreta las regularidades (o patrones) del discurso cotidiano que encarnan durante la formación docente, determinadas inscripciones en el cuerpo/sujeto de los actores alumnos. Su propósito fue analizar el discurso de los estudiantes y descifrar los significados encarnados que circulan en las prácticas de formación.

Para realizar su análisis se basaron en la acepción de poder desarrollada por Foucault que lo concibe como una relación de fuerzas, sostiene que el poder no es una propiedad que se posee sino una estrategia que se ejerce por medio del discurso histórico.

Para realizar el análisis del discurso de los alumnos recortaron lo que denominaron un "campo discursivo", entendido este como una estructura institucional dinámica constituida por las relaciones discursivas que operan en ese espacio heterogéneo que habitan los actores involucrados. 


\section{REFERENCIAS}

Alarcón, L. y Conde, J. (2003): Manuales Escolares, ciudadanía e identidad nacional en el Caribe colombiano. Proyecto de Investigación a COLCIENCIAS. Mayo. Universidad del Atlántico. Barranquilla. Colombia.

Argibay,M.; Celorio, G. y Celorio, J. (1991): La cara oculta de los textos escolares. Investigación curricular en Ciencias Sociales. Servicio Editorial Universidad del país Vasco. Bilbao.

Audigier, F. et al. (1991): "La place de savoirs scientifiques dans le didactiques de I'historie et le geographie". En Revue Francaise de Padagogie. № 106.

Audiguier, F. (1984): "Articulation eccole/college. Etude diagnostique". En Rapport Historie-Geographie. París: INRP.

Bernete, F. (1994): "Cómo analizar las representaciones sociales contenidas en los libros de texto de Historia". Comunicación, Lenguaje y Educación. № 22. Universidad Complutense de Madrid. Madrid. España.

Bini, G.; Calero, M.; Luque, G.; et al. (1977): Los libros de texto en América Latina. Editorial Nueva imagen. México.

Blanch, J. (2008): "Enseñar a Enseñar Historia". Miradas a la Historia. No 7. Universidad Autonóma de Barcelona. España.

Bralich, J. (1990): Los textos escolares como instrumento ideológico. Universidad de la República. Departamento de Publicaciones. Montevideo. Uruguay.

Braslavsky, C. (1994): "Los usos de la Historia en los libros de texto para las escuelas primarias argentinas (1916-1930)". Cuadernillos FLACSO. No 144. Alemania.

Braslavsky, C. (2007): La enseñanza de la Historia. Los Libros de texto en su contexto: Argentina 1975-1989 (Compilación). Organización de Estados Americanos. Ciudad de México. México.

Briolo, J. y Herrera, H. (2003): Las intenciones del docente y la progresión del discurso áulico. Producciones Científicas: Sección Educación y Ciencias Sociales. Universidad de Catamarca. Colombia.

Busani, M. (2006):: El cuerpo/sujeto y el poder en el aprendizaje de la práctica profesional. Ponencia presentada en el Congreso de lingüística de la Universidad Nacional del Comahue. Viedma. Río Negro. Argentina.

Cabrera, J. (2003): "Discurso docente en el aula". Estudios Pedagógicos. No 29. Universidad Austral de Chile. Chile. 
Campagnucci, et al. (2002): "Acerca de las prácticas docentes y la enseñanza de la psicología". Revista de Teoría y didáctica de las Ciencias Sociales. Mérida-Venezuela. Enero-Diciembre. № 7.

Candela, A. (2001): "Corrientes teóricas sobre discurso en el aula". Revista Mexicana de Investigación Educativa. Mayo-Agosto. Vol. 6, núm. 12, pp. 317-333

Chiroque, S. (2007): Juicios sobre Fujimori en los textos escolares. Informe del Instituto de Pedagogía Popular. No 68. Lima. Perú.

Cubas, D. (1996): Contenido Ideológico de los textos escolares primarios de Historia Nacional (1890-1910). U.N.C Facultad de filosofía y letras. Mendoza. Argentina.

De Longhi, A. (2000): El discurso del profesor y del alumno: análisis didáctico. Investigación Didáctica. Enseñanza de las Ciencias. Textos. Córdova. Argentina.

Domínguez, M. y Varela, C. (2008): "El discurso escolar de la ciudadanía: una mirada desde la hermenéutica". Revista Electrónica de la Enseñanza de la Ciencia. Volumen 7. Mérida. Venezuela.

Downe y Levstik (1991): "Teaching and learning History". En Shaver, J.: Haandbook of research of social studies teaching and learning. New York MacMillan Publishing Company.

Eiros, N. (1996): Los estudiantes y los textos históricos: una relación conflictiva. Clio \& Asociados: La Historia Enseñada. No 1. Universidad Nacional del Litoral. Santa Fe. Argentina.

Fontaine, L. y Eyzaguirre, B. (1997): "¿Por qué es importante el texto escolar?" En: El futuro en riesgo: nuestros textos escolares. Centro de Estudios Públicos. Santiago de Chile. Chile.

Galván, L. (2000): Actividades y Resultados del Seminario: "Libros Escolares Mexicanos, siglos XVIII, XIX y XX". Centro de Investigaciones y Estudios Superiores en Antropología Social. México.

Galván, L. (2005): "Héroes, antihéroes y la sociedad mexicana: en los libros de texto de Historia (1994-1997)". En: Los procesos Independentistas iberoamericanos en los manuales de Historia. Volumen I. Países Andinos-España. Fundación Mapfre. Tavera. Organización de Estados Iberoamericanos. Madrid. España.

Garcia, N. (2000): Textos Escolares y Patrimonio Cultural. CNCR. Editorial Dibam. Santiago de Chile. Chile.

Giraldo, L. (2009): Caracterización del discurso pedagógico del docente e identificación de los actos de habla. CENDA. Bogotá-Colombia. 
Grinberg, S. y Palermo, A. (2000): Mujeres y Trabajo en los textos escolares: Crisis y Perspectivas. Educere. Octubre-Diciembre. Año/Vol 4. Número 011. Universidad de los Andes. Mérida. Venezuela.

Guanipa, M. y Velazco, L. (2005): Hermenéutica del discurso del profesor. Lingua Americana. Año IX No 17. Chile.

Gvirtz, S.; Larripa, S. y Oría, A. (2005): "Prácticas discursivas pedagógicas, didácticas y escolares: algunas categorías para repensar la relación entre el saber y la escuela". Revista de Pedagogía. Universidad de San Andrés. Argentina.

Herrera, M.; Pinilla, A. y Suaza, M. (2003): La identidad nacional en los textos escolares de ciencias sociales. Colombia 1900-1950. Universidad Pedagógica Nacional. Bogotá. D.C.

Lacan, J. (1988): El Seminario. Libro 2. Paidós.

Jarquín, G. (2006): Práctica docente alternativa: un acercamiento desde el ACD. Universidad Complutense de Madrid. Espéculo. № 30. Madrid. España.

Jiménez, P. y Díaz. (2003): Discurso de Aula y Argumentación: Consideraciones Teóricas y Metodológicas. Investigación Didáctica. Universidad de Santiago de Compostela. España.

Kalafunja, M. y Obeleagu, A. (2002): "Un estudio sobre la interacción en el aula de las escuelas de educación primaria". Perspectivas, Vol. XXXII, Marzo. UNESCO.

Kauffmann, C. (2001): "Producciones sobre textos escolares argentinos: hitos, tendencias y potencialidades". En: Historia de la Educación. Anuario No 4. 2001-2002. Sociedad Argentina de Historia de la Educación. Argentina.

Linuesa, M. (2006): Los sistemas de valores en los textos escolares: un modelo de análisis. Facultad de Filosofía y Ciencias de la Educación. Salamanca. España.

Llobera, M. (1990): "Reconsideración del discurso interactivo". Comunicación, Lenguaje y Educación. Núm. 7 Revista de Didáctica ELE.

Maristany, J. (1988): Las ficciones del pasado en los manuales de historia argentina (1880-1910). Publicaciones de la Universidad Nacional de la Pampa. Argentina.

Martínez-Otero, V. (2004): "La Calidad del discurso educativo: análisis y regulación a través de un modelo pentadimensional". Revista Complutense de Educación. Vol. 15. Núm. 1. Universidad Complutense de Madrid.

Mendoza, M. (2006): La perspectiva discursiva en el análisis historiográfico del libro escolar. Memoria, conocimiento y utopía. Número 2. Otoño. México. 
Monroy, B. (1993): "Análisis educativo del texto escolar". Educación y Cultura. № 31. Bogotá. Colombia.

Morales, O. y Lischinsky, A. (2008): "Discriminación a través de las ilustraciones de libros de texto de Educación Secundaria Obligatoria en España". Discurso y Sociedad. ISSN 1887-4606. Vol. 2 (1) 115-152. Barcelona. España.

Muscará, F. (1999): Contenido Ideológico en los textos escolares. Publicaciones del consejo de Investigaciones de la Universidad Nacional del Cuyo. Mendoza. Argentina.

Ossenbach, G. (2000): "La Investigación sobre los manuales escolares en América Latina". En Historia de la Educación. Revista Interuniversitaria. Salamanca. № 19. España. Pp 195-203.

Peter, J. (1991): "The Professional craft knowledge of History Teacher". En: Teaching History. The Historical Association. № 64. Reino Unido.

Ramirez, T. (2002): "El texto escolar como objeto de reflexión e investigación". Docencia Universitaria Vol. III, año 2002, No 1. U.C.V .Caracas. Venezuela.

Ramírez, T. (2007): "Los textos escolares en Venezuela 1958-2004"'". Sapiens. Revista Universitaria de Investigación. Año 8. № 2. Caracas. Venezuela.

Riekenberg, et al. (2000): Latinoamérica: Enseñanza de la Historia, libros de texto y conciencia histórica. Alianza Editorial. FLACSO. Venezuela.

Romero, E. (1994): El discurso de la Patria: La Historia de Chile en la sala de clases (1843-1880). Tesis para optar al grado de Licenciado en Historia. Pontificia Universidad Católica de Chile. Instituto de Historia. Santiago de Chile. Chile.

Sánchez, E. (2008): "Elementos para analizar la interacción entre estudiantes y profesores". Revista de Educación. № 346. Mayo-Agosto. Caracas. Venezuela.

Searle, J. (1986): Actos de Habla. Cátedra. Madrid.

Simancas, K. (1998): "Algunos Criterios para el Análisis de textos escolares de Geografía". Geo-enseñanza. Vol. Especial. Universidad de los Andes. Táchira. Venezuela.

Souto, X. (2002): "Los Manuales Escolares y su influencia en la instrucción escolar". Revista Bibliográfica de Geografía y Ciencias Sociales. ISSN: 1138-9796. Vol. VII. No 414. Diciembre. Universidad de Barcelona. España.

Tarabay, F. y León, A. (2007): "La argumentación como forma de comunicación en el discurso del profesor universitario". Acción Pedagógica. № 16. Enero-Diciembre pp. 136. 
Tiana, A. (1999): "La investigación histórica sobre los manuales escolares en España". En: La Historia Enseñada. Clio \& Asociados. № 4. Pp. 101-119. Barcelona. España.

Tiana, A. (2000): El libro escolar, reflejo de intenciones políticas e influencias pedagógicas. Universidad Nacional de Educación a Distancia. Paseo de la Castellana. Madrid. España.

Torrealba, M. (2004): "La interacción docente y el discurso pedagógico de la ironía". Revista Educere. Año 8. № 26. Mérida. Venezuela.

Valls, R. (2001): "Los nuevos retos de las investigaciones sobre los manuales escolares de historia: entre textos y contextos". Revista de Teoría y Didáctica de las Ciencias Sociales. No 6. ISSSN 1316-955. Mérida. Venezuela.

Viladot, L.; Gómez, I. y Malagarriga, T. (2008): "Análisis del discurso en un aula de música". Revista electrónica Complutense de Investigación en Educación musical. Volumen 5, Número 3. Tomado de: http://www.ucm.es/info/reciem el 30-03-2010.

Weiss, K. (1978): Report of the 1977 national survey of sciencie and social studies education. En: Research Triangle Park. N.C. Center for educational research and evaluationas campañas políticas son las que hacen al triunfo o derrota de un político en las elecciones. Por eso, cada detalle es pensado de manera minuciosa para que nada falle a la hora de expresarle a la ciudadanía que un candidato determinado es el indicado para representarla.

\section{María Elena Del Valle de Villalba}

Graduada de Profesora en la Universidad Pedagógica Libertador en el año 1992, con especialización en Historia Económica y Social de Venezuela en la Universidad Santa María, con Maestría en Historia Económica y Social de Venezuela. Doctora en Ciencias de la Educación en el Pedagógico de Caracas. Profesora Ordinaria de la Universidad Pedagógica Libertador. Coordinadora del Núcleo de Investigaciones Geohistóricas Lisandro Alvarado, cuenta con proyectos financiados por la UPEL y con participación de la Universidad Complutense de Madrid, Universidad de Barcelona-España, Universidad de las Palmas de Gran Canaria y Universidad Pedagógica de Colombia. Investigadora certificada de la Universidad Complutense de Madrid y del CELARG. Ponente regular en eventos nacionales e internacionales, adscrita a la línea de investigación Ciencias del lenguaje, y con siete años de investigación en el área de análisis del discurso. Investigadora acreditada del CYET. 